\title{
PERSPECTIVE
}

\section{Infection as a comorbidity of COPD}

\author{
S. Sethi
}

ABSTRACT: The normal lung has several defence mechanisms to deal with microorganisms. Lower respiratory infections in the absence of lung disease are therefore relatively infrequent as compared with upper respiratory tract infections in healthy adults. In the setting of chronic obstructive pulmonary disease (COPD), lower respiratory tract infections, both acute and chronic, occur with increased frequency. As these infections contribute considerably to the clinical course of the patient with COPD, they constitute a significant comorbidity in COPD. Recurrent acute infections by bacterial and/or viral pathogens are now clearly linked with the occurrence of exacerbations of COPD. In addition, the occurrence of pneumonia in COPD has received considerable recent attention as it appears to be increased by the use of inhaled corticosteroids. The role of chronic infection in the pathogenesis of COPD is an active area of research with several different types of pathogens potentially implicated. Additionally, COPD patients with HIV infection have a more rapidly progressive decline in lung function than non-HIV-infected patients. Enhanced understanding of the host-pathogen interaction is needed to better prevent and treat respiratory tract infection in COPD.

KEYWORDS: Chronic obstructive pulmonary disease, community-acquired pneumonia, HIV, infection

$\mathrm{H}$ istorically, the term "comorbidity" has been used to indicate a medical condition existing simultaneously with but independently of another condition. Recently, however, use of the term comorbidity has broadened to suggest a reciprocal or causal relationship between two disease states. Certainly, in the case of chronic obstructive pulmonary disease (COPD), this perspective is intriguing from both an academic and a clinical perspective. It is likely that infection has a larger role than currently recognised in the pathogenesis of COPD, and the relationship between the two can be viewed as a complicated comorbid one, which may affect both the direction and course of each problem. The present article reviews the potential contribution of bacterial infection as a comorbid condition to the aetiology, pathogenesis and clinical course of COPD. Table 1 lists bacterial pathogens implicated in acute and chronic infections in COPD.

\section{INFECTION CYCLES IN COPD}

Under normal conditions, the healthy human tracheobronchial tree and lung parenchyma have a remarkable ability to maintain sterility, in spite of repetitive exposure to microbial inocula from micro-aspiration and inhalation. In the setting of
COPD, however, this innate lung defence appears to be disrupted as a result of exposure to smoke and other environmental irritants. The impairment in lung defence results in two distinct infection cycles in COPD that could contribute to progressive loss of lung function (fig. 1).

The acute cycle is well recognised. The lung in COPD becomes susceptible to repeated acute airway mucosal infections with viruses and bacterial pathogens, leading to episodes of increased inflammation and worsened symptoms, which are clinically diagnosed as exacerbations of COPD.

The less well-recognised infection cycle in COPD is the chronic cycle, whereby microbial colonisation results in chronic inflammation and lung destruction, conceptualised as the "vicious circle hypothesis" [1]. This hypothesis posits that once impaired innate lung defence due to tobacco smoking allows microbial pathogens to become established in the lower respiratory tract, the microbial pathogens further impair mucociliary clearance and lung defence due to increased mucus secretion, disrupted ciliary activity and airway epithelial injury [1]. Thus, microbial colonisation of the lower airways in patients with
AFFILIATION

Division of Pulmonary, Critical Care and Sleep Medicine, Dept of Medicine, University at Buffalo, State University of New York, Buffalo, NY, USA.

\section{CORRESPONDENCE}

S. Sethi

Division of Pulmonary, Critical Care and Sleep Medicine, Dept of

Medicine, University at Buffalo State University of New York VA Western New York Health Care System

3495 Bailey Avenue Buffalo NY 14215 USA

E-mail: ssethi@buffalo.edu

Received:

May 212009

Accepted after revision: Oct 292009 
TABLE 1 Bacterial pathogens implicated in acute and chronic infections in chronic obstructive pulmonary disease

\begin{tabular}{lcc} 
Microbe & Role in exacerbations & Role in stable disease \\
\hline Bacteria & & Major pathogen \\
Haemophilus influenzae & $20-30 \%$ of exacerbations & Minor role \\
Streptococcus pneumoniae & $10-15 \%$ of exacerbations & Minor role \\
Moraxella catarrhalis & $10-15 \%$ of exacerbations & Undefined \\
Pseudomonas aeruginosa & $5-10 \%$ of exacerbations, prevalent in advanced disease & Unlikely \\
Enterobacteriaceae & Isolated in advanced disease, pathogenic significance undefined & Unlikely \\
Haemophilus haemolyticus & Isolated frequently, unlikely cause & Unlikely \\
Haemophilus parainfluenzae & Isolated frequently, unlikely cause & \\
Staphylococcus aureus & Isolated infrequently, unlikely cause & Commonly detected, pathogenic significance undefined \\
Atypical bacteria & & Unlikely \\
Chlamydophila pneumoniae & $3-5 \%$ of exacerbations & $1-2 \%$ of exacerbations
\end{tabular}

COPD can perpetuate itself. Furthermore, this chronic presence of bacteria in the lower airways is not innocuous; rather, it induces inflammation and can contribute to progressive airflow obstruction and lung damage characteristic of this disease [1].

\section{ROLE OF INFECTION IN COPD EXACERBATIONS: A HISTORICAL PERSPECTIVE}

Recurrent acute exacerbations secondary to infections by bacterial and/or viral pathogens are seen in almost all patients with COPD. Although exacerbations were previously perceived as a nuisance problem with no serious consequences, there is now ample evidence that they contribute to poor quality of life, mortality and progression of lung disease in COPD [2]. Furthermore, exacerbations have been shown to account for $35-45 \%$ of the healthcare costs for COPD, as they lead to both in-patient and outpatient healthcare utilisation [3].

For decades, researchers explored the role of bacterial infection in COPD exacerbations, yet consensus remained elusive. Early studies based on culture of respiratory secretions and serological tests revealed that about one-third of exacerbations were related to viral infection. However, in the remaining

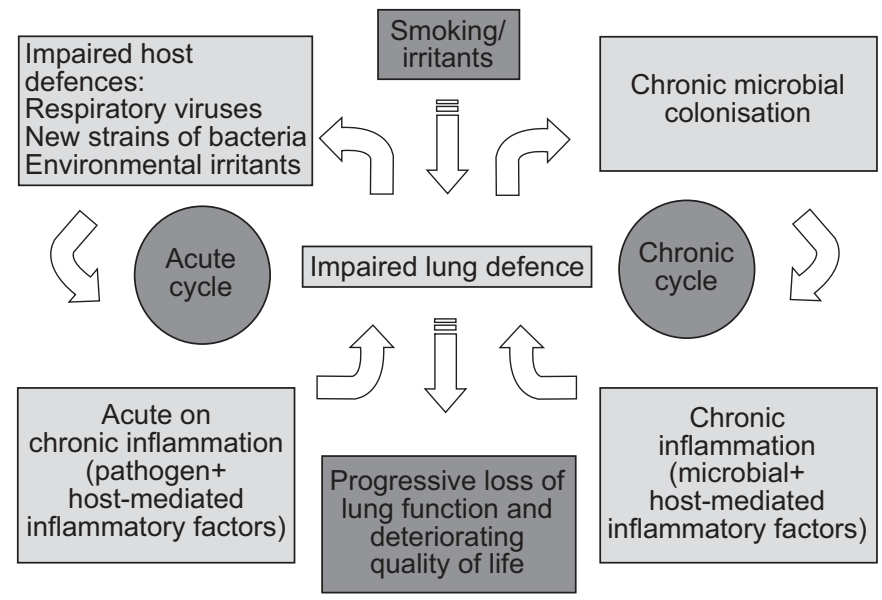

FIGURE 1. Two distinct infection cycles in chronic obstructive pulmonary disease. cases, the aetiology appeared to be uncertain; specifically, the significance of bacterial isolation from sputum was unclear [4]. A causal relationship between bacteria and exacerbation was largely discredited when longitudinal studies showed similar rates of isolation of Streptococcus pneumoniae and Haemophilus influenzae from sputum during both acute exacerbations and stable disease [4-8]. A significant limitation of these studies was their failure to differentiate among strains within a bacterial species [9], as the technology was not available at that time for such differentiation. Consequently, changes in strains within a species in the airways of individual patients over time were undetected.

In recent years, new research techniques, including molecular typing, have led to renewed interest in the area of bacteria and COPD. Recent studies have used more sophisticated diagnostic tools and the application of molecular technologies to further explore a causal relationship between infection and exacerbation.

\section{MECHANISMS OF BACTERIAL PATHOGENESIS IN COPD}

In order to explain bacterial pathogenesis of exacerbations in spite of similar frequency of bacterial isolation during stable disease and exacerbations, it was previously quite popular to apply a bacterial load model for the pathogenesis of acute exacerbations attributing increased bacterial concentration in the lower airways as a cause of exacerbation [10, 11]. This hypothesis, however, was not well supported by empirical data.

An alternative model that is now well supported with empirical evidence is based on the premise that acquisition of new bacterial strains from the environment is the major driver of exacerbations (fig. 2) [12]. In this model, following acquisition of a new bacterial strain, the balance between the virulence of the pathogen and the host lung defence determines the degree of excess airway and systemic inflammation engendered by the bacterial pathogen. The degree of increased inflammation in turn determines the extent of symptoms, which, if they lead to the patient seeking healthcare, are diagnosed as an acute exacerbation. The host mounts an adaptive immune response to the infecting bacterial strain, which, possibly augmented by the use of antibiotics, 


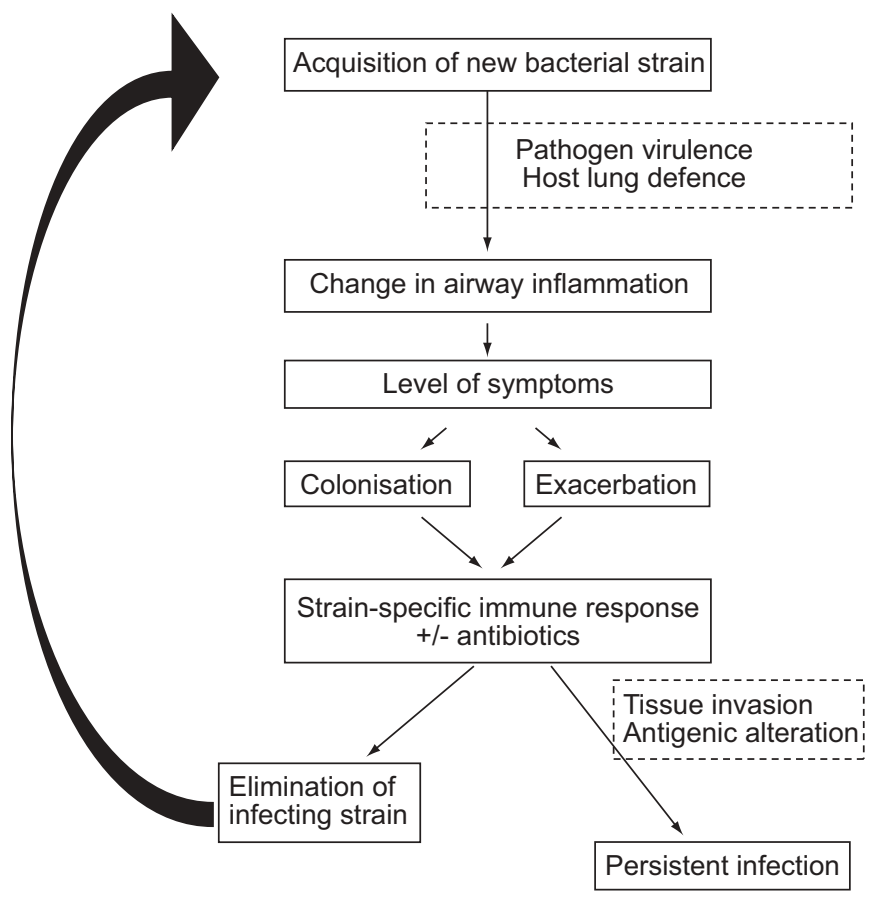

FIGURE 2. Current model of pathogenesis of bacterial exacerbation in chronic obstructive pulmonary disease. Reproduced and modified from [12], with permission from the publisher.

controls and eventually eliminates the infecting strain. However, because the immune response is strain-specific, antigenically unrelated strains from the same species and of course other bacterial species cause recurrent exacerbations.

Much of the evidence to support this model of exacerbation pathogenesis comes from an ongoing prospective cohort study in COPD in my own centre (VA Medical Center at Buffalo, Buffalo, NY, USA). A cohort of 50 patients with COPD was initially enrolled in 1994, with additional enrolment as needed to maintain the size of the cohort. These patients were seen monthly and whenever they had symptoms suggestive of an exacerbation [9]. At each visit, clinical information, and sputum and serum samples were collected. Molecular typing was performed on strains of non-typeable H. influenzae, S. pneumoniae, Moraxella catarrhalis and Pseudomonas aeruginosa collected over 6-10 yrs [9]. The study found that the frequency of exacerbations increased more than two-fold at clinic visits at which a new strain with one of these four major pathogens was isolated from sputum (fig. 3a) [9]. Statistically significant increased risk of exacerbations was seen with $H$. influenzae, $S$. pneumoniae and $M$. catarrhalis acquisition.

Recently, we have extended these observations. A proportion of the isolates initially identified as variant $H$. influenzae in the ongoing COPD study previously referred to, were later characterised as non-haemolytic Haemophilus haemolyticus [13]. A re-examination of the relationship between $H$. influenzae strain acquisition and exacerbation after excluding the $H$. haemolyticus strains showed a four-fold increase in the incidence of exacerbation associated with such acquisition [13]. Re-examination of the relationship between acquisition of new strains of $P$. aeruginosa with COPD exacerbation after the
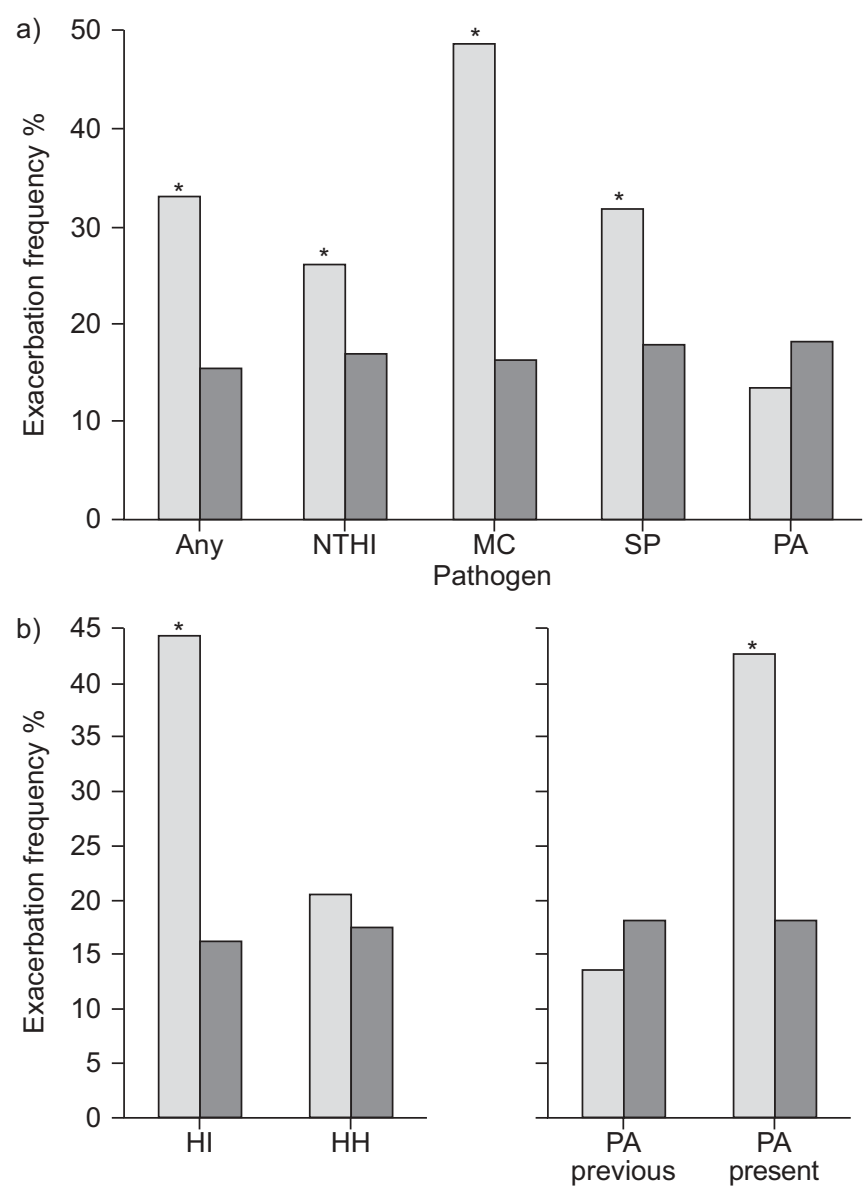

FIGURE 3. a) New strain isolation and exacerbation frequency. b) Updated data regarding the relationship of new strain acquisition and exacerbation frequency for Haemophilus influenzae $(\mathrm{HI})$, Haemophilus haemolyticus $(\mathrm{HH})$ and Pseudomonas aeruginosa (PA). NTHI: non-typeable HI; MC: Moraxella catarrhalis; SP: Streptococcus pneumoniae. Relative risk $(95 \% \mathrm{Cl})$ of exacerbation in a) were: any 2.15 (1.83-2.63); NTHI 1.69 (1.37-2.09); MC 2.96 (2.39-3.67); SP 1.77 (1.142.75); PA $0.61(0.21-1.82)$. Relative risk ( $95 \% \mathrm{Cl}$ ) of exacerbation in b) were: $\mathrm{HI} 4.09$ (2.89-5.80); PA: 3.36 (1.88-6.03). *: $p<0.05$. a) Based on data reported by SETHI et al. [9]. b) Based on data reported by MURPHY and co-workers [13, 14].

addition of 4 years of data to our original data set revealed that such acquisition did indeed increase the risk of exacerbation by more than three-fold (fig. 3b) [13, 14].

If isolation of new bacterial strains at exacerbation is indeed causal, then a distinct inflammatory profile should be seen with these exacerbations when compared with exacerbations without new strains. When airway and systemic inflammation during new strain exacerbations are compared with exacerbations without new bacterial strains, a greater intensity of neutrophilic airway inflammation and systemic inflammation is demonstrated in the former group [15]. Furthermore, WHITE et al. [16] have shown that clearance of bacteria following 10 days of treatment with antibiotics for purulent sputum exacerbations was associated with a reduction in neutrophilic inflammation, while bacterial persistence was associated with persistent elevation of airway inflammation.

The contribution of bacterial load to the occurrence of exacerbations of COPD is uncertain. To explore this question, 
additional analysis of the ongoing COPD study was undertaken to determine whether increased bacterial concentrations function as a separate mechanism for exacerbation induction, independent of new strain acquisition. Among pre-existing strains, no differences in sputum concentrations of $H$. influenzae and $H$. haemolyticus were reported during exacerbation compared with stable disease [17]. Concentrations of Haemophilus parainfluenzae were significantly lower during exacerbations than during stable periods, and S. pneumoniae trended similarly. Among new strains of $M$. catarrhalis and $H$. influenzae, however, increased concentrations of $\sim 0.5 \log$ were in fact observed during exacerbation, as compared with during stable visits [17]. Although statistically significant, this small difference in bacterial load is unlikely to be biologically significant [18]. The findings demonstrate that exacerbations are not a function of increased bacterial concentration of preexisting bacterial strains. The small increases in concentrations of new bacterial strains during exacerbation that were observed in this study may be a function of host-pathogen interaction and are unlikely to be an independent mechanism for exacerbations of COPD for the pathogens included in that study [17]. However, Enterobacteriaceae, P. aeruginosa and Staphylococcus aureus have been implicated in COPD exacerbations and were not systematically examined in this study; therefore, bacterial load changes could be a mechanism of exacerbations for these pathogens. Bacterial load is also an important determinant of airway inflammation, with increasing concentrations associated with greater intensity of neutrophilic airway inflammation $[19,20]$.

Another approach to examining the role of bacteria during exacerbations is to study the development of specific immune responses to bacterial pathogens following exacerbations. Such specific immune responses to infecting strains of $H$. influenzae, S. pneumoniae and $M$. catarrhalis have been demonstrated, either systemic (serum immunoglobulin (Ig)G) and/or mucosal (sputum $\operatorname{Ig} \mathrm{A}$ ), particularly when the strain is a new acquisition. Furthermore, for $H$. influenzae, this immune response has considerable strain specificity [21-23], allowing antigenically disparate strains of this species to cause recurrent exacerbations.

Recent studies have also demonstrated that co-infection with virus and bacteria is seen in about a quarter of exacerbations, and these episodes tend to be of greater clinical and physiological severity [24, 25].

\section{ANTIBIOTIC TREATMENT OF EXACERBATIONS}

Clarification of the role of bacteria in exacerbations of COPD, meta-analyses of randomised placebo-controlled trials and recent epidemiological studies support the use of antibiotics to treat selected exacerbations of COPD [26-28]. Current guidelines recommend the use of antibiotics for moderate and severe exacerbations (i.e. those requiring hospitalisation or characterised by at least two of the three cardinal symptoms of increased dyspnoea, sputum volume and/or sputum purulence) $[26,29]$. In mild exacerbations, which do not meet the severity criteria above, withholding antibiotics and treating symptomatically is recommended [26, 29].

Rather than using the same antibiotic for all exacerbations as initial empiric therapy, a stratification approach to choosing an antibiotic that takes into account risk factors for poor outcome and the probability of infection with an antimicrobial-resistant pathogen is currently advocated [26, 29]. Patients with exacerbations can be stratified into complicated (those with one or more of the following risk factors: advanced age, severe airflow obstruction, frequent exacerbations, comorbid cardiac disease) or uncomplicated (none of those risk factors). Complicated patients should be treated with a fluoroquinolone or amoxicillin/clavulanate, whereas for uncomplicated patients, a macrolide, cephalosporin, tetracycline or trimethoprim/sulfamethoxazole is appropriate. In all patients, a class of antibiotics that differs to those received in the previous 3 months for any reason should be used, in order to minimise ineffective therapy because of antibiotic resistance. Response to therapy should be evaluated at 48-72 h.

\section{COMMUNITY-ACQUIRED PNEUMONIA IN COPD}

A major cause of hospitalisation and a common cause of death [30], community-acquired pneumonia (CAP) is most commonly seen in individuals who smoke cigarettes and/or have COPD [31]. However, controversy exists as to whether the prognosis of pneumonia is altered by pre-existing COPD.

A population-based case-control study of CAP assessed that the population-attributable risk of tobacco smoking on the development of CAP in adults was 32\% [32]. Ex-smokers were reported to have a reduction of $\sim 50 \%$ in CAP risk after 5 yrs of tobacco-smoking cessation; after 10 yrs, the risk was comparable to lifelong nonsmokers [32]

Concerns have been raised that the use of inhaled corticosteroids, which are commonly used to treat COPD, may increase the risk for CAP. This was most apparent in the large TORCH (TOwards a Revolution in COPD Health) study, which compared inhaled fluticasone, salmeterol and combined salmeterol/fluticasone to placebo over 3 yrs [33]. In this study, both the inhaled steroid-containing arms were associated with increased incidence of pneumonia, with a doubling of the incidence in the combination arm from 4 to $8 \%$ over the $3-y r$ period. A meta-analysis of 11 studies of combination preparations (fluticasone/salmeterol and budesonide/formoterol) has confirmed these findings, with an OR 1.83 (95\% CI 1.51-2.21) increase in the overall risk for pneumonia attributed to the combination treatment [34]. In a retrospective database analysis, the effect appeared to be dose-related, with doses $>1,000 \mathrm{mcg} \cdot \mathrm{day}^{-1}$ of beclomethasone equivalent more likely to be linked to pneumonia than lower doses. One cautionary area in the interpretation of these findings is that in most instances, a clinical diagnosis of pneumonia was made without radiographic confirmation. It is possible that some instances of reported pneumonia were in fact severe bacterial exacerbations characterised by purulent sputum, fever and/or localised physical findings, leading the clinician to a diagnosis of pneumonia.

The mechanism of this adverse effect of inhaled steroid use in COPD is not well understood. In a pathologic study of resected lungs from patients with very severe COPD, inhaled steroid use was associated with a reduction in the number of lymphoid follicles in small airways. One can speculate that this represents blunting of the immune response to bacterial infection in the distal lung, altering the host-pathogen balance 
in airways, thereby increasing susceptibility to bacterial infections presenting as CAP.

The impact of underlying COPD on the outcome of CAP is at present unclear. Some retrospective studies of patients with COPD have reported increased mortality risks [35, 36] but others have not $[37,38]$. Mechanisms that may contribute to poor CAP outcome in COPD include the presence of resistant bacteria, which were perhaps not properly treated from the outset, as well as reduced lung reserve. Factors that could actually decrease mortality from CAP in COPD include prior immune experience with related bacterial pathogens that may have protective effects, enhanced airway inflammation, and the use of systemic corticosteroids acutely for treatment of the concomitant "exacerbation" [38].

COPD has been observed to alter the pathogens associated with CAP. Although the pneumococcus still remains predominant, an increased incidence of $H$. influenzae and occasionally $M$. catarrhalis is seen. The presence of very severe COPD with concomitant bronchiectasis and repeated courses of antibiotics predisposes these patients to pneumonia caused by $P$. aeruginosa [39]. However, aetiological diagnosis of pneumonia becomes more difficult in COPD because chronic colonisation confounds the interpretation of sputum culture results. COPD and smoking also increase serum titres to Chlamydia pneumoniae, rendering serological diagnosis for this infection difficult to interpret, especially when single titres are used for diagnosis.

\section{CHRONIC INFECTION IN COPD}

Microbial pathogen presence in the airways of patients with COPD has been regarded as "colonisation", primarily because of the absence of acute symptoms of infection. However, the appropriate definition of colonisation is the presence of a pathogen that does not cause damaging effects to the host or elicit a host response. In contrast, several recent studies show that bacterial pathogens are associated with host inflammatory and immune responses in stable COPD [40-46]. These findings suggest that application of the term colonisation to the lower airway bacterial presence in COPD is a misnomer, because it is likely that it is a low-grade chronic infection, with significant pathophysiological consequences. Various microbial pathogens have been implicated in chronic infection in COPD. These include typical bacteria such as non-typeable $H$. influenzae and $P$. aeruginosa, atypical bacterium such as $C$. pneumoniae, viruses such as adenovirus and possibly respiratory syncytial virus, and recently a fungus, Pneumocystis jiroveci.

The vicious circle hypothesis is a useful conceptual model showing how chronic infection can contribute to COPD progression (fig. 1). Evidence supporting this hypothesis is accumulating, such as the demonstration of a microbial colonisation-associated lower airway neutrophilic inflammation, as discussed below. Additional support for this hypothesis comes from the demonstration of persistent inflammation in ex-smokers with COPD, pathological evidence of local adaptive immune responses in the small airways in COPD, and radiological evidence of bronchiectasis developing in advanced disease [40]. Chronic microbial infection can contribute to inflammation in COPD as a direct inflammatory stimulus or indirectly by altering the host response to tobacco smoke.
More than 40 yrs ago, LAURENZI et al. [41] demonstrated the first protected specimen brushings to reveal pathogens in the lower respiratory tract of patients with COPD and chronic bronchitis, in contrast to sterile lung environments in healthy nonsmokers. BANDI et al. [42] used in situ hybridisation and immunofluorescence microscopy to demonstrate intracellular $H$. influenzae in bronchial mucosal biopsies of eight out of 24 patients with clinically stable COPD.

In longitudinal sputum samples obtained from patients in our COPD study clinic, we observed gaps in the presence of strains of $H$. influenzae in sputum by culture. Molecular detection, however, established that strain-specific $H$. influenzae DNA was detected in the majority of the sputum samples that had yielded negative cultures, demonstrating persistent infection [43]. Similar observations have now been made for $P$. aeruginosa, implying that sputum cultures underestimate the frequency of bacterial colonisation in COPD.

\section{COLONISATION AS AN INFLAMMATORY STIMULUS IN STABLE COPD}

Several studies have demonstrated that colonization of the tracheobronchial tree could serve as an inflammatory stimulus in the airway and potentially contribute to disease progression [44]. An observational study of 67 subjects with clinically stable moderate-to-severe COPD revealed that those with potentially pathogenic microorganisms (PPMs) in their sputum exhibited higher neutrophil differential counts, greater sputum supernatant levels of interleukin (IL)-8, leukotriene $B_{4}$, tumour necrosis factor (TNF)- $\alpha$ and neutrophil elastase, and a more exaggerated neutrophil chemotactic response than the noncolonised subjects [45]. In another study, bronchoalveolar lavage (BAL) was used to determine the pattern of airway inflammation in smokers and patients with stable COPD, and its relation to bronchial microbial colonisation [46]. Eight nonsmoking and 18 smoking controls as well as 52 patients with COPD were studied. $40 \%$ of the smoking controls and a third of the COPD subjects were colonised in the bronchial tree. When colonised subjects from both groups were compared to noncolonised subjects, the presence of PPMs was significantly associated with bronchial neutrophilia and higher TNF- $\alpha$ concentration [45].

In order to further test the hypothesis that bacterial colonisation is associated with airway inflammation in stable COPD, BAL was obtained in three groups of subjects: 26 ex-smokers with stable COPD, 20 ex-smokers without COPD (ex-smokers), and 15 healthy nonsmokers (nonsmokers) [44]. Quantitative bacterial cultures, cell counts, chemokine, cytokine, proteinase/antiproteinase, and endotoxin levels in the BAL fluid were compared [44]. PPMs were recovered in $34.6 \%$ of COPD, $0 \%$ of ex-smokers, and $6.7 \%$ of nonsmokers. Colonised COPD subjects had significantly greater relative and absolute neutrophil counts, IL-8, active matrix metalloproteinase- 9 and endotoxin levels in the BAL than the noncolonised COPD subjects. Several inflammatory constituents of BAL were also significantly elevated in colonised COPD subjects when compared with ex-smokers and nonsmokers. These results further demonstrate that bacterial colonisation drives inflammation of the distal airways in patients with COPD [44]. 
Latent adenovirus infection has been shown to exaggerate the inflammatory response to tobacco smoke, both in lung tissue specimens and in an animal model [47]. With sensitive molecular detection techniques, the presence in lung tissue of several other viruses, such as respiratory syncytial virus, Epstein-Barr virus and other microbial pathogens (Pneumocystis and Chlamydia, for example), has been demonstrated. The significance of these observations is as yet undefined but these pathogens could play a role in COPD pathogenesis in selected patients.

\section{CHRONIC INFECTION AND COPD PROGRESSION}

Although the rate of decline in lung function following smoking cessation returns to that of a non-smoker in patients with early COPD, this may not be the case with more advanced disease. As discussed above, persistent airway inflammation in ex-smokers is observed in relation to microbial colonisation. Whether this microbial colonisation-induced inflammation contributes to COPD disease progression is not clear, though some suggestive evidence exists. In a small study of 30 patients with advanced COPD, WILKINSON et al. [48] observed that an increase in airway bacterial load over the 1-yr follow-up was related to a decline in forced expiratory volume in $1 \mathrm{~s}$ (FEV1). Additional larger studies are required to confirm and extend these observations.

\section{ANTIBIOTIC USE IN STABLE COPD}

Prophylactic or suppressive antibiotic treatment of bacterial infection in COPD was attempted several decades ago and is once again emerging as a therapeutic option [49]. In studies performed prior to 1970 , there was a statistically significant benefit to such treatment, but it was small and because of concerns of antibiotic resistance, such treatment was not recommended [49]. Ongoing studies are examining intermittent treatment with inhaled and oral fluoroquinolones and chronic treatment with low-dose macrolides in patients with COPD and repeated exacerbations. Results of these studies will determine whether such treatment is effective and safe.

\section{ROLE OF HIV INFECTION IN COPD}

Several investigations suggest that HIV infection is an independent risk factor for the development of COPD [50]. An intriguing link between HIV and COPD is infection with Pneumocystis. The frequency of Pneumocystis colonisation rises with increasing severity of airflow obstruction in COPD, suggesting a possible pathogenic link [51]. In addition, there have been several reports that HIV-seropositive people develop an accelerated form of emphysema, an effect that is compounded in HIV-positive patients with a history of smoking [52]. Additional mechanisms undoubtedly contribute to the observed association between HIV and COPD. These include a high rate of smoking in the HIV-positive population, the presence of HIV in the lung, increased oxidative stress related to the viral load, and recurrent pneumonia. The degree of immunosuppression and use of antiretroviral therapy may also impact on development or progression of COPD in HIV disease [50].

\section{CONCLUSION}

Respiratory infection, both acute and chronic, is likely to have a larger role than currently recognised in the pathogenesis and course of COPD. COPD is heterogeneous; therefore, the role of infection may vary among patients with this disease. It is also likely to be more significant in the airway (bronchitis, bronchiolitis) than in the parenchymal (emphysema) component of COPD. Infection and COPD appear to have a reciprocal and causal relationship, thereby denoting infection as a comorbid condition in COPD. Predisposition to infection in COPD is partly related to smoking but appears to worsen when airflow obstruction develops in smokers. Enhanced understanding of the host-pathogen interaction in the setting of COPD will lead to new strategies for the prevention of exacerbations and novel interventions for the prevention of COPD progression.

\section{SUPPORT STATEMENT}

The manuscript was developed from presentations and discussions at the Comorbidities in COPD Task Force, held in March 2008 in Jacksonville, FL, USA. Responsibility for the content rests with the author, who originated and developed the intellectual content and performed substantive scientific revisions. The meeting, author participation, and manuscript preparation were supported by Boehringer Ingelheim and Pfizer. Boehringer Ingelheim and Pfizer did not review or edit the manuscript except to check it for medical misrepresentation.

\section{STATEMENT OF INTEREST}

A statement of interest for S. Sethi can be found at www.erj.ersjournals. $\mathrm{com} / \mathrm{misc} /$ statements.dtl

\section{ACKNOWLEDGEMENTS}

The author would like to acknowledge the editorial assistance of G. Belfiglio from Advanced Studies in Medicine (Somerville, NJ, USA), whose services were provided by Boehringer Ingelheim and Pfizer.

\section{REFERENCES}

1 Sethi S, Murphy TF. Bacterial infection in chronic obstructive pulmonary disease in 2000: a state-of-the-art review. Clin Microbiol Rev 2001; 14: 336-363.

2 Mannino DM, Homa DM, Akinbami LJ, et al. Chronic obstructive pulmonary disease surveillance - United States, 1971-2000. MMWR Surveill Summ 2002; 51: 1-16.

3 Andersson F, Borg S, Jansson SA, et al. The costs of exacerbations in chronic obstructive pulmonary disease (COPD). Respir Med 2002; 96: 700-708.

4 Gump DW, Phillips CA, Forsyth BR, et al. Role of infection in chronic bronchitis. Am Rev Respir Dis 1976; 113: 465-474.

5 McHardy VU, Inglis JM, Calder MA, et al. A study of infective and other factors in exacerbations of chronic bronchitis. $\mathrm{Br} J$ Dis Chest 1980; 74: 228-238.

6 Smith $\mathrm{CB}$, Golden $\mathrm{C}$, Klauber MR, et al. Interactions between viruses and bacteria in patients with chronic bronchitis. J Infect Dis 1976; 134: 552-561.

7 Fagon JY, Chastre J. Severe exacerbations of COPD patients: the role of pulmonary infections. Semin Respir Infect 1996; 11: 109-118.

8 Tager I, Speizer FE. Role of infection in chronic bronchitis. N Engl J Med 1975; 292: 563-571.

9 Sethi S, Evans N, Grant BJ, et al. New strains of bacteria and exacerbations of chronic obstructive pulmonary disease. $N$ Engl J Med 2002; 347: 465-471.

10 Gompertz S, O'Brien C, Bayley DL, et al. Changes in bronchial inflammation during acute exacerbations of chronic bronchitis. Eur Respir J 2001; 17: 1112-1119. 
11 Stockley RA, O'Brien C, Pye A, et al. Relationship of sputum colour to nature and outpatient management of acute exacerbations of COPD. Chest 2000; 117: 1638-1645.

12 Veeramachaneni SB, Sethi S. Pathogenesis of bacterial exacerbations of COPD. COPD 2006; 3: 109-115.

13 Murphy TF, Brauer AL, Sethi S, et al. Haemophilus haemolyticus: a human respiratory tract commensal to be distinguished from Haemophilus influenzae. J Infect Dis 2007; 195: 81-89.

14 Murphy TF, Brauer AL, Eschberger K, et al. Pseudomonas aeruginosa in chronic obstructive pulmonary disease. Am J Respir Crit Care Med 2008; 177: 853-860.

15 Sethi S, Wrona C, Eschberger K, et al. Inflammatory profile of new bacterial strain exacerbations of chronic obstructive pulmonary disease. Am J Respir Crit Care Med 2008; 177: 491-497.

16 White AJ, Gompertz S, Bayley DL, et al. Resolution of bronchial inflammation is related to bacterial eradication following treatment of exacerbations of chronic bronchitis. Thorax 2003; 58: 680-685.

17 Sethi S, Sethi R, Eschberger K, et al. Airway bacterial concentrations and exacerbations of chronic obstructive pulmonary disease. Am J Respir Crit Care Med 2007; 176: 356-361.

18 Wilkinson TM. Host pathogen interaction during COPD exacerbations: moving on from microbiology by numbers? Am J Respir Crit Care Med 2007; 176: 323-325.

19 Sethi S, Muscarella K, Evans N, et al. Airway inflammation and etiology of acute exacerbations of chronic bronchitis. Chest 2000; 118: 1557-1565.

20 Hill AT, Campbell EJ, Hill SL, et al. Association between airway bacterial load and markers of airway inflammation in patients with stable chronic bronchitis. Am J Med 2000; 109: 288-295.

21 Chapman AJ Jr, Musher DM, Jonsson S, et al. Development of bactericidal antibody during Branhamella catarrhalis infection. J Infect Dis 1985; 151: 878-882.

22 Faden H, Bernstein J, Brodsky L, et al. Otitis media in children. I. The systemic immune response to nontypable Hemophilus influenzae. J Infect Dis 1989; 160: 999-1004.

23 Yi K, Sethi S, Murphy TF. Human immune response to nontypeable Haemophilus influenzae in chronic bronchitis. J Infect Dis 1997; 176: 1247-1252.

24 Wilkinson TM, Hurst JR, Perera WR, et al. Effect of interactions between lower airway bacterial and rhinoviral infection in exacerbations of COPD. Chest 2006; 129: 317-324.

25 Papi A, Bellettato CM, Braccioni F, et al. Infections and airway inflammation in chronic obstructive pulmonary disease severe exacerbations. Am J Respir Crit Care Med 2006; 173: 1114-1121.

26 Sethi S, Murphy TF. Infection in the pathogenesis and course of chronic obstructive pulmonary disease. $N$ Engl J Med 2008; 359: 2355-2365.

27 Ram FS, Rodriguez-Roisin R, Granados-Navarrete A, et al. Antibiotics for exacerbations of chronic obstructive pulmonary disease. Cochrane Database Syst Rev 2006; 2: CD004403.

28 Roede BM, Bresser P, Bindels PJ, et al. Antibiotic treatment is associated with reduced risk of a subsequent exacerbation in obstructive lung disease: an historical population based cohort study. Thorax 2008; 63: 968-973.

29 Global Initiative for Chronic Obstructive Lung Disease (GOLD). Global strategy for the diagnosis, management and prevention of chronic obstructive pulmonary disease. www.goldcopd.org/ GuidelinesResources.asp?11=2\&12=0: Date last updated: 2007 . Date last accessed: January 2008.

30 Pneumonia and influenza death rates: United States; 1979-1994. MMWR Morb Mortal Wkly Rep 1995; 44: 535-537.

31 Ginesu F, Pirina P. Etiology and risk factors of adult pneumonia. J Chemother 1995; 7: 277-285.
32 Almirall J, Gonzalez CA, Balanzo X, et al. Proportion of community-acquired pneumonia cases attributable to tobacco smoking. Chest 1999; 116: 375-379.

33 Calverley PM, Anderson JA, Celli B, et al. Salmeterol and fluticasone proprionate and survival in chronic obstructive pulmonary disease. N Engl J Med 2007; 356: 775-789.

34 Nannini L, Cates CJ, Lasserson TJ, et al. Combined corticosteroid and long-acting $\beta$-agonist in one inhaler versus placebo for chronic obstructive pulmonary disease. Cochrane Database Syst Rev 2007; 4: CD003794.

35 Rello J, Rodriguez A, Torres A, et al. Implications of COPD in patients admitted to the intensive care unit by communityacquired pneumonia. Eur Respir J 2006; 27: 1210-1216.

36 Restrepo MI, Mortensen EM, Pugh JA, et al. COPD is associated with increased mortality in patients with community-acquired pneumonia. Eur Respir J 2006; 28: 346-351.

37 Ruiz De Ona JM, Gomez Fernandez M, Celdran J, et al. [Pneumonia in the patient with chronic obstructive pulmonary disease. Levels of severity and risk classification.] Arch Bronconeumol 2003; 39: 101-105.

38 Garcia-Vidal C, Calbo E, Pascual V, et al. Effects of systemic steroids in patients with severe community-acquired pneumonia. Eur Respir J 2007; 30: 951-956.

39 American Thoracic Society/European Respiratory Society. Standards for the Diagnosis and Management of Patients with COPD. www.thoracic.org/sections/copd/resources/copddoc.pdf Date last updated: 2004. Date last accessed: July 30, 2009.

40 Hogg JC, Chu F, Utokaparch S, et al. The nature of small-airway obstruction in chronic obstructive pulmonary disease. $N$ Engl $J$ Med 2004; 350: 2645-2653.

41 Laurenzi GA, Potter RT, Kass EH. Bacteriologic flora of the lower respiratory tract. $N$ Engl J Med 1961; 265: 1273-1278.

42 Bandi V, Apicella MA, Mason E, et al. Nontypeable Haemophilus influenzae in the lower respiratory tract of patients with chronic bronchitis. Am J Respir Crit Care Med 2001; 164: 2114-2119.

43 Murphy TF, Brauer AL, Schiffmacher AT, et al. Persistent colonization by Haemophilus influenzae in chronic obstructive pulmonary disease. Am J Respir Crit Care Med 2004; 170: 266-272.

44 Sethi S, Maloney J, Grove L, et al. Airway inflammation and bronchial bacterial colonization in chronic obstructive pulmonary disease. Am J Respir Crit Care Med 2006; 173: 991-998.

45 Banerjee D, Khair OA, Honeybourne D. Impact of sputum bacteria on airway inflammation and health status in clinical stable COPD. Eur Respir J 2004; 23: 685-691.

46 Soler N, Ewig S, Torres A, et al. Airway inflammation and bronchial microbial patterns in patients with stable chronic obstructive pulmonary disease. Eur Respir J 1999; 14: 1015-1022.

47 Vitalis TZ, Kern I, Croome A, et al. The effect of latent adenovirus 5 infection on cigarette smoke-induced lung inflammation. Eur Respir J 1998; 11: 664-669.

48 Wilkinson TM, Patel IS, Wilks M, et al. Airway bacterial load and FEV1 decline in patients with chronic obstructive pulmonary disease. Am J Respir Crit Care Med 2003; 167: 1090-1095.

49 Black P, Staykova T, Chacko E, et al. Prophylactic antibiotic therapy for chronic bronchitis. Cochrane Database Syst Rev 2003; 1: CD004105.

50 Crothers K. Chronic obstructive pulmonary disease in patients who have HIV infection. Clin Chest Med 2007; 28: 575-587.

51 Morris A, Sciurba FC, Lebedeva IP, et al. Association of chronic obstructive pulmonary disease severity and Pneumocystis colonization. Am J Respir Crit Care Med 2004; 170: 408-413.

52 Diaz PT, King MA, Pacht ER, et al. Increased susceptibility to pulmonary emphysema among HIV-seropositive smokers. Ann Intern Med 2000; 132: 369-372. 